\title{
LA TEORÍA HISTÓRICO-CULTURAL DE VYGOTSKI DESDE UNA PERS- PECTIVA FENOMENOLÓGICA
}

\author{
VYGOTSKI'S CULTURAL-HISTORICAL THEORY FROM A PHENOMENO- \\ LOGICAL PERSPECTIVE
}

\author{
Jorge Montesó Ventura \\ UNED(España) \\ jordimonteso@gmail.com
}

\begin{abstract}
Resumen: Mucho se ha discutido sobre la idoneidad de situar a Vygotski en el Olimpo de la psicología. Todavía hoy, 80 años después, sus teorías siguen despertando tantos partidarios, defensores de su originalidad, como efusivos detractores que abrazan las insuficiencias metodológicas de sus trabajos. Una de las insuficiencias que, a nuestro juicio, más ha acusado es la falta de una sólida fundamentación teórica que dote de sentido el gran cúmulo de datos y resultados de que dispone. Por ello, el objetivo del presente artículo es mostrar una factible salida que pasaría por el encuentro, reencuentro en ocasiones, con ciertas proposiciones fenomenológicas, para el caso, desde los estudios fenomenológicos de la percepción y su atención al peso de los predicados culturales, donde el lenguaje se erige como elemento principal y, a la vez, punto de unión con Vygotski, lo que ayudará a reforzar los fundamentos de su propuesta.
\end{abstract}

Palabras clave: Vygotski, psicología históricocultural, fenomenología, percepción.

\begin{abstract}
Many times we have discussed about the appropriateness of situating to Vygotski in the Olympus of psychology. Even today, 80 years later, his theories continue creating as many supporters, defenders of his originality, as effusive critics who accuse the methodological shortcomings of his work. In our view, one of the shortcomings that have accused more his work is the lack of a solid theoretical foundation that endows meaning with the available host of data and results. Therefore, the objective of this article is to show a feasible solution that would be to approach, sometimes encounter with certain phenomenological propositions, for that matter, from the phenomenological studies of perception and its attention to the weight of cultural predicates, where the language is the main element and, at the same time, the meeting point with Vygotski which will help strengthen the foundations of its proposal.
\end{abstract}

Keywords: Vygotski, cultural-historical psychology, phenomenology, perception. 
Recientemente revisaba el que, con toda probabilidad, fue uno de los últimos artículos que el profesor Siguán publicase en vida. ${ }^{1}$ El artículo, bajo el título "Relectura de Luria", eran unas reflexiones, con tintes de reconocimiento, sobre el alcance que tuvo para la psicología española el descubrimiento de Vygotski y, con él, de su teoría histórico-cultural. ${ }^{2}$ De facto, el artículo se convertía en una especie de llamada de atención sobre la actualidad que todavía hoy pudiera mantener, o al menos debiera, la teoría histórico-cultural dado el interés que sigue despertando la comprensión del ser humano en tanto ser social o cultural. Teniendo en cuenta que, como decía el propio Siguán $-y$ aquí compartimos-, "el problema principal para el estudio científico del comportamiento humano es explicar cómo un sujeto puramente biológico [...] se convierte a la vez en subjetivo y en social" (Siguán 2010, 57), el pensamiento vyogotskiano, con sus aciertos e inconvenientes, destinado fundamentalmente a responder a semejante problema, debería situarse en una relativa centralidad dentro de los estudios del comportamiento humano. Sin embargo, pese al reconocimiento prestado por el profesor, el artículo no escondía cierto halo melancólico en sus conclusiones. $Y$ es que, en ocasiones, los inconvenientes pesan más que los aciertos aun pudiendo ser estos más leves o coyunturales, pero cuando las ambiciones son elevadas, y las de Vygotski sin duda lo fueron, el juicio no suele ser menos severo. ${ }^{3}$ El caso es que la actual situación del pensamiento vygotskiano, comparado con el de otros modelos, no parece alcanzar ese lugar que desearía el profesor Siguán -aunque mantuvo una esperanza que hoy alentamos-, pues el interés general de la psicología, si es que hay algo que se pueda llamar así, parece no querer anidar sobre él, un hecho que, a nuestro humilde juicio, entre otras faltas, evidencia cierto despiste, sostenido, en las ciencias del comportamiento para con sus asuntos.

El principal reto de Vygotski, al que alude el profesor y que desglosaremos en este artículo, fue dar razón de cómo la sociedad ejerce de elemento coexistente al sujeto, en síntesis y sin exclusiones, en la determinación de su

\footnotetext{
${ }^{1}$ Hablamos de "Relectura de Luria". Revista de historia de la psicología, núm. 33, vol. 1, 2010, pp. 5164. Publicado en marzo de 2010 , solo dos meses antes de su fallecimiento.

2 Sobre cómo se introdujeron las obras de Vygotski en España: García, Luciano. N. "La 'canonización' de Vigotski en España y Argentina (1978-1991)". Revista de historia de la psicología, núm. 36, vol. 2, 2015, pp. 7-30; para testear su impacto: VV.AA. Actualidad de Lev. S. Vygotski. Siguán, Miguel (coord.). Barcelona: Anthropos, 1987.

3 Para una visión crítica sobre la originalidad vygotskiana: Perinat, Adolfo. "Mis desencuentros y mi reencuentro con Vygotsky". Quaderns de Psicologia, núm. 11, vol. 1/2, 2009, pp. 51-60.
} 
comportamiento. Estudiar semejante vínculo prestaría la comprensión de los mecanismos subyacentes a todo acto a la vez que ofrecería una comprensión más integral del comportamiento humano, un comportamiento que, por ser socialmente modulado, definiría al agente como un sujeto intencional y proyectivo. Algo tan elemental como esto es lo que el profesor Siguán llevaba siguiendo y analizando desde que descubriese a Vygotski a finales de los sesenta, tan elemental que, hoy día y salvando algunas diferencias, a nadie le costaría conectar con gran parte de las ideas fenomenológicas vigentes. $Y$ en ello yace la justificación de nuestro artículo: en ese intento por establecer y reforzar puentes de unión entre las ideas vygotskianas y la fenomenología, así como la idea de avivar una corriente, la histórico-cultural, que, bien conectada con otras escuelas, podría desatar un conjunto de sinergias que facilitasen una necesaria y más amplia comprensión del comportamiento humano, motivo para el que se postuló.

Un acercamiento comparativo semejante lo esbozó, en 1996, Igor Klyukanov. En un artículo sobre las semejanzas entre los estudios fenomenológicos y la psicolingüística, apuntaba a ciertos puentes de conexión entre ambas disciplinas que, necesariamente, implicaban las ideas vygostkianas. Klyukanov hablaba de la intencionalidad de la conciencia defendida por la fenomenología y su similitud con la idea de mediación semiótica en Vygotski; aludía al paralelismo dado en la dicotomía existente entre conciencia pre-reflexiva y reflexiva con las funciones mentales elementales y complejas del ruso; la intersubjetividad como elemento necesario para el reconocimiento de la propia humanidad en cada sujeto; o la relación entre cuerpo y lenguaje que nos conduce a hablar de un lenguaje contextualizado, esto es, históricamente aprehendido. Como vemos, los puentes son importantes y de substancial recorrido, tal que el propio autor cerraba su artículo afirmando que "los esfuerzos fenomenológicos y psicolingüísticos deberían considerarse como perspectivas diferentes del mismo objeto, que le añaden densidad" (Klyukanov 1996, 327).

Pues en esa labor de añadir densidad ubicamos el presente artículo. Labor a la que debemos añadir los trabajos de MacDonald (2000) en los que fundamentó históricamente la influencia de Husserl en el pensamiento del psicólogo ruso. Para MacDonald, esta se sustenta en dos puntos fundamentales $(2000,70)$ : la teoría de la parte y el todo defendida en Investigaciones lógicas; y el carácter pre-teorético y descriptivo de la psicología husserliana. De la primera defiende 
(Ibid., 91) que Vygostki siempre mantuvo "la idea fundamental de que todas las experiencias perceptivas destapan 'objetos' [...] dentro de un marco horizontal [...] y que las transformaciones de estas totalidades [perceptivas] pueden tener lugar independientemente de cualquier cambio en los 'objetos'". De la segunda, alega que "para la antropología crítica, el método fenomenológico reveló un mundo pre-teórico de la física ingenua y relaciones prelógicas [...] no susceptibles de explicación o de clasificación en términos racionales, científiconaturales" (MacDonald 2000, 91), idea de la Vygotski se vio fuertemente impregnado.

Tal influencia, comenta MacDonald (2000, 76-77), alcanzó al ruso, en primer lugar, a través de las teorías lingüísticas de Jakobson - con quien Husserl mantuvo estrechos vínculos a través del Circulo Lingüístico de Praga ${ }^{4}-$ como también mediante la obra de Gustav Shpet, quien fuera alumno de Husserl en Gotinga y, después, profesor de Vygotski en Moscú. Otra fuente vendría a través de los seminarios que Chelpanov, director del Instituto de Psicología moscovita, organizó entre 1915 y 1916 en los que se abordó la fenomenología husserliana ${ }^{5}$ y el análisis de las ideas de Koffka, donde el moravo se halla muy presente (Ibid., 77). Otras vías indirectas a las que alude MacDonald son, precisamente, la simpatía del ruso por los teóricos de la forma, a través de los cuales recibió fuertes dosis de fenomenología; la antropología de Lévy-Bruhl, quien marcó mucho el pensamiento filogenetista del ruso; y, finalmente, por los trabajos de psicología infantil de Piaget (Ibid., 80), todos ellos, autores con reconocidas influencias husserlianas.

Las huellas de tal influjo las podemos hallar fundamentalmente en Pensamiento y lenguaje y en La crisis de la psicología (MacDonald 2000, 88 y sig.). En el primero, se observan en el despliegue de las aplicaciones de la teoría de la parte y el todo en los estudios gestálticos; también cuando alude a la idea del "habla interna" y de los dos planos del lenguaje -fonético y semántico-, que retomaremos en adelante y que albergan muchas resonancias con la fenomenología más allá de Husserl. En el segundo texto, vemos cómo Vygotski resume las tres tendencias, para él dominantes, en la psicología coetánea si-

\footnotetext{
${ }^{4}$ Holenstein, Elmar. "Jakobson and Husserl". Human Context, núm. 7, 1975, pp. 61-83.

${ }^{5}$ MacDonald (2000, 77-78) comenta cómo el propio Vygotski, en su abordaje de la crisis de la psicología, criticó las distorsionadas interpretaciones que Chelpanov realizó de la obra husserliana.
} 
guiendo un esquema que recuerda mucho al esbozado por Husserl en "La filosofía como ciencia estricta", el de las etapas conceptuales situadas históricamente en la evolución de las ideas científicas naturales.

Recientemente, otro estudio (Veer y Yasnitski 2015) ha puesto a la vista otro nexo entre la fenomenología y Vygotski que ya se advertía con bastante facilidad en sus lecturas: el papel de Erich Jaensch. Los autores reparan en las distintas discusiones que el ruso mantuvo sobre las ideas del breslavo sugiriendo incluso que ambos podrían haber mantenido correspondencia, con lo que el conocimiento de Vygotski sobre fenomenología, con todo lo dicho, queda más que acreditado.

Dicho lo cual, en el presente artículo, para no rehacer caminos, nos centraremos en un aspecto diferencial que nos permita ampliar los lazos de unión entre ambas corrientes: los estudios perceptivos. Fundamentalmente nos interesa esta vía por entender que la percepción es una de las ocupaciones más elementales en todo ser vivo, una ocupación que se sitúa, dado su estado de primaria disposición, en la base de toda conducta humana, incluso de la lingüística y, por tanto, un buen modelo para dar razón de todo tipo de comportamiento. Dentro de la percepción, el análisis de atención y su capacidad selectiva resultará crucial, pues posibilita que dicha percepción sea, en todo caso, consciente y, por mediación de los intereses que la mueven, históricamente circunscrita, culturalmente modulada, lo que nos acercará mucho a las teorías del ruso. Este, pues, será el contenido del presente artículo y la aportación que realicemos en ese incremento de densidad conectiva.

\section{LA TEORÍA HISTÓRICO-CULTURAL}

Sobre la teoría histórico-cultural y los postulados generales de Vygotski se ha escrito ya suficientemente como para pretender aportar nada insólito. Fácilmente nos podemos remitir a los propios trabajos de Vygotski o Luria -que se defienden por sí mismos-, o a las revisiones críticas de René van der Veer (2007), Anton Yasnitsky (2016), o los trabajos bibliográficos de Ekaterina Zavershneva (2010). En nuestro caso nos limitaremos a exponer, someramente, los postulados fundamentales de su teoría para encuadrar el siguiente apartado, centrado en el análisis de la percepción, desde el que daremos el salto a la fenomenología. 
Así diremos que, lo que fundamentalmente pretendió Vygotski, fue ofrecer una explicación, lo más integral posible, del comportamiento humano sin caer en las parcialidades de algunas corrientes psicológicas del momento que, bien por su exacerbado idealismo, bien por su empirismo radical, se mostraban incompletas para consumar la deseada explicación. ${ }^{6}$ Dada su pretensión de compleción, Vygotski no podía adherirse a ninguna de ellas, pero tampoco renunciar a las mismas; así, quizá influenciado por los teóricos de la forma, entendió que ambas corrientes debían estar presentes en síntesis dialéctica para una adecuada explicación del comportamiento. ${ }^{7}$ Por ello, para el ruso, el contexto social en el que vive el sujeto humano, a través de sus usos culturales, resultaría tan importante como su propia biología para explicar su modus operandi y, a través de él, su modus essendi. Por este motivo, sus trabajos se encaminaron a dar razón científica de cómo el ser humano transita de ese ser que en su génesis es meramente biológico a un ser que se define culturalmente, esto es, "explicar cómo un individuo fisiológico se convertía en una realidad social" (Siguán 2010, 57). Él mismo afirmaba que la historia de la conducta del niño nace a partir de la interrelación de procesos elementales, de origen biológico, y las funciones psicológicas superiores, de origen sociocultural (cfr. Vygotski y Luria 2007)..$^{8}$ Tal síntesis, en esencia, se daba mediante la adquisición del signo como herramienta, esto es, mediante la apropiación e interiorización del lenguaje. Según Vygotski, el sujeto humano va recorriendo, desde su nacimiento hasta su adultez, un proceso de socialización o "desarrollo histórico" en el que el habla se adquiere e interioriza hasta vincularse, tras una serie de cambios cuantitativos y cualitativos, al pensamiento. Ello sucede a partir de los dos años de

\footnotetext{
${ }^{6}$ Lo explicita el propio Vygotski en su comunicación al Congreso Nacional de Psiconeurología de Leningrado, en enero de 1924. Cfr. Vygotski, Lev S. "El método de investigación reflexológica y psicológica". Problemas de la psicología contemporánea, núm. 2, 1930, pp. 26-46.

${ }^{7}$ Vygotski sentía simpatía por las teorías de Köhler y Lewin siendo muy probable que fuese a través de ellos que retomara algunas posiciones de raíz marcadamente fenomenológica. Cfr. Yasnitsky, Anton. "A transnational history of "the beginning of a beautiful friendship"the birth of the cultural-historical Gestalt psychology of Alexander Luria, Kurt Lewin, Lev Vygotsky, and others". En Yasnitsky, Anton y van der veer, René (Eds.) Revisionist Revolution in Vygotsky Studies. Londres: Routledge, 2016, pp. 201-226.

${ }^{8}$ Aprovechamos la primera cita del autor para advertir las necesarias precauciones que se deben mantener cuando abordemos sus obras, fundamentalmente a la hora de otorgar validez a su autoría. Existen muchas obras atribuidas al autor que son fruto de compilaciones posteriores o bien obras marcadamente censuradas por el gobierno soviético. Para conocer tales avatares, resulta imprescindible leer: Van der Veer, René y Yasnitsky, Anton. "Vygotsky in English: What still needs to be done". Integrative Psychological and Behavioral Science, núm. 45, vol. 4, 2011, pp. 475- 493; van der veer, René y Yasnitsky, Anton. "Vygotsky the published. Who wrote Vygotsky and what Vygotsky actually wrote". En Yasnitsky, Anton y Van der Veer, René (Eds.) Revisionist Revolution in Vygotsky Studies. Londres: Routledge, 2016, pp. 73-93.
} 
edad, cuando "las líneas de desarrollo del pensamiento y el habla, que hasta el momento avanzaban separadamente, se cruzan, coinciden y dan comienzo a una forma completamente nueva de comportamiento, solo característica del hombre" (Vygotski y Luria 2007, 146), una conducta paulatinamente más elaborada que irá posibilitando nuevos procesos mentales, los llamados "superiores". $^{9}$

Estos estudios, de evolución ontogenética, fueron complementados en Vygotski por otros de traza filogenética, trabajos que se acercaron a los antropológicos de Lévy-Bruhl o a la sociología de Thurnwald y que gravitaron sobre los estudios de Köhler, Bühler, Stern o el propio Engels. En ellos, Vygotski defendió que el desarrollo cultural del ser humano, en tanto especie, venía acompañado de un mayor control o domino de sus procesos cognitivos, donde el elemento crítico de progreso era el uso del habla, el modo en que esta interrelacionaba con el pensamiento: ${ }^{10}$ "La diferencia específica entre la operación realizada por el niño y la propia de los animales superiores estriba en que este campo atencional [...] selecciona mediante el habla, de entre todos los elementos del campo perceptivo, aquellos que compondrán propiamente el «campo futuro.»" (Vygotski y Luria 2007, 44-45).

Así mismo, defendía - lo que le valió, y sigue valiendo, de centro de numerosas críticas- que, como sucede a nivel filogenético, el niño '"recapitula' en su socialización-educación los pasos (algunos) que ha efectuado la humanidad en su progreso cultural" (Perinat 2007, 24), un progreso en sus capacidades que se da gracias a esa interrelación cultura-conciencia desembocando en el ser cultural que es el sujeto.

En sus axiomas, Vygotski puso siempre el acento en la aparición del lenguaje como momento crítico dentro del proceso de crecimiento humano, y a nuestro juicio no herró en su dictamen, sin embargo, como ya hemos apuntado, a nosotros nos interesará ponerlo en una esfera diferente aunque, como el propio Vygotski afirmase, complementaria al uso lingüístico: la percepción. "La unidad de percepción, lenguaje y acción, que conduce a la reconstrucción de las

\footnotetext{
${ }^{9}$ Cuando aludamos a la distinción entre procesos elementales y superiores, debemos tener en cuenta que, a partir de los años 30, el propio Vygotski cuestionó la radicalidad de tal diferenciación, optando por un enfoque más sistémico e integral, siendo las funciones superiores un dominio de las inferiores (cfr. Zavershneva, 2010, 42).

${ }^{10}$ Los estadios por los que, según Vygotski, transita el niño en su interiorización del habla los despliega el ruso en su obra Pensamiento y habla.
} 
leyes del campo visual, constituye el auténtico y vital objeto de análisis de la investigación dirigida a estudiar el origen de las formas específicamente humanas de comportamiento" (Vygotski y Luria 2007, 23).

El elemento perceptivo se erige, pues, junto al lenguaje, como fundamental para la comprensión de la acción; una acción que entendemos en cuanto ocupación, como gesto planificado destinado a alcanzar un fin, un objeto; un gesto poseedor de sentido, tan significativo para el desarrollo humano que es capaz de definir su ser como un ser-proyectivo que tiende-a (atiende) un mundo cultural del que forma parte y com-parte, su mundo vivido.

\section{LA PERCEPCIÓN EN LOS PLANTEAMIENTOS VYGOTSKIANOS}

Lo que inicialmente apremió a Vygotski en sus estudios sobre percepción fue remarcar la singularidad del proceso perceptivo humano frente al seguido por otras especies animales. Ello le ofrecería, debido al carácter elemental de la percepción, argumentos sólidos para justificar lo distintivo del comportamiento humano en su conjunto. Para él, la percepción humana no era el mero desarrollo continuo y directo de la percepción animal en una escala evolutiva, sino que, en su despliegue, se producía un salto cualitativo, una diferenciación esencial que marcaba la particularidad de dicha percepción. Aislar el elemento responsable de semejante salto era su principal objetivo y, este, como ya intuía por sus trabajos precedentes, subyacía en el uso del lenguaje.

Vygotski coincidía con Köhler ${ }^{11}$ en que la percepción era un elemento esencial y determinante para la comprensión del comportamiento de todo ser, humano o no. Ahora bien, a diferencia del humano, al animal parecía faltarle un ingrediente en la ecuación que, según él, explicaba el comportamiento - percepción-lenguaje-acción-, faltaba el ingrediente central. En el animal, sin la presencia del elemento lingüístico, percepción y acción se enlazan de manera inmediata, siendo el comportamiento resultante un acto directamente motivado por el objeto o entorno percibido. En términos orteguianos diríamos que, a diferencia del ser humano, el animal vive "alterado", "agarrado con la atención al escenario cósmico" (OC. VIII, 322). El ser humano, por el contrario, añadiendo

${ }^{11}$ Köhler, Wolfgang. "Zur Psychologie des Schimpansen". Aus der Anthropoidenstation auf Teneriffa, vol. 5. Sitzungsberichte der Preußischen Akademie der Wissenschaften, 1921. 
a la unidad el elemento lingüístico, lo cambia todo, pues desde una etapa muy temprana del desarrollo, y a diferencia del animal, "el niño comienza a percibir el mundo no solo a través de sus ojos, sino también a través del habla" (Vygotski y Luria 2007, 38), percibe a través de conceptos y, por ello, consigue descubrir el mundo a través del sentido de las cosas, de su logos. Desde muy temprano, el niño percibe su entorno no como un agregado de meras impresiones, sino como algo que para él posee sentido, que es comprensible y alberga significado. La realidad como tal se percibe a través de un conjunto de estructuras de significación posibilitadas, precisamente, por el uso de conceptos, de palabras, esto es, no me limito a percibir el mundo de un modo fisiológico como haría un chimpancé, sino que además lo hago de un modo biográfico, histórico, a través de predicados culturales, mediante ese acervo conceptual que obtengo de mi comunidad en mi crecimiento. Así, decía Vygotski que el proceso de "interiorización" de las formas culturales de comportamiento:

está vinculado con cambios profundos en la actividad de las funciones psíquicas más importantes y con la reconstrucción de la actividad psíquica sobre la base de operaciones con signos. Por una parte, dejan de existir los procesos psíquicos naturales tales como los vemos en los animales, para ser incorporados a este sistema de comportamiento ahora reconstruido desde una base psico-cultural de modo que constituye una entidad nueva [que] debe incluir las anteriores funciones elementales que siguen existiendo de forma subordinada (Vygotski y Luria 2007, 69).

Esa "interiorización" de elementos culturales, fundamentalmente del habla, es la base del salto cualitativo entre el humano y el resto de animales. Gracias al uso del lenguaje, el sujeto consigue, entre otros avances, percibir objetos reales, percibe el mundo en tanto lo vive, mediante estructuras significativas. Veremos en el siguiente apartado las numerosas analogías que, en este aspecto, se dan con los estudios fenomenológicos de la percepción, especialmente con los de Schapp y Ortega.

Un paso más en los estudios perceptivos lo realizó el ruso a través de los procesos de selección. Comenzamos a especificar el papel activo, y necesario, de la atención en la percepción humana, del que Vygotski no dudó en afirmar que "por la importancia de su papel en este tipo de operaciones debería concederse a la atención el primer lugar entre estas funciones" (Vygotski y Luria 2007, 44).

Vygotski analizó cómo el niño, en un proceso de toma de decisiones, no actuaba como lo hace un adulto. El niño seleccionaba a la par que actuaba, esto 
es, el gesto atencional siempre iba acompañado del gesto activo de sus manos, del movimiento de su cuerpo como conjunto. En el niño, percepción y acción aún se dan muy próximas, casi paralelamente. En el adulto, empero, esta vinculación se ve dilatada, la atención se distancia del movimiento posibilitando que el sujeto se atienda a sí mismo, abriendo un tiempo para la reflexión previa a la acción. Si, como advertíamos, en el animal se aprecia una directa relación entre percepción-acción, vemos cómo en el niño esta pronto comienza a distanciarse hasta tomar, en el adulto, una disociación ostensible. Ello es debido, según Vygotski, a la aparición de funciones psicológicas complejas en el interfaz entre percepción-acción como son el pensamiento o la reflexión, o lo que es lo mismo, el uso interiorizado de signos. Lo que sucede, afirmaba, es que "al despegarse el movimiento de la percepción directa y al someterse a las funciones simbólicas incorporadas al acto reactivo, se rompe con la historia natural de la conducta y se pasa a una nueva página: la de la actividad intelectual superior del ser humano" (Vygotski y Luria 2007, 43).

En definitiva, lo que parece suceder es que, con la ayuda del lenguaje, de una creciente capacidad de interpretar lo percibido, el ser humano comienza a comprender y a dominar su cuerpo, sus reacciones, también su atención, domina su mente en general y aprende a dirigirla a su voluntad hasta poder desvincularla del espacio vivido. Frente al campo visual, el ser humano es capaz de crear un campo temporal - potencial e imaginario- que, para él, resulta tan perceptible y real como el primero; un campo creado mediante reflexión, mediante pensamiento; un campo que, a diferencia del meramente visual, le permite planificar la acción a desempeñar. Por ello, un modo fundamental de definir al ser humano es como "ser proyectivo", pues mediante ese gesto de abstracción, la conducta humana deja de estar únicamente determinada por el contexto, como sucede con el resto de animales, y nos permite añadir la evaluación y planificación de nuestra acción. A diferencia, pues, del animal que, como diría Ortega, siempre vive "alterado", el humano es capaz de flexionar su atención sobre sí mismo, distanciarse del entorno perceptivo y "ensimismarse" para concebir la acción futura. Así, si el mono necesita ver para atender, el humano $-y$ esto es lo específicamente humano- puede prestar atención para ver (cfr. Vygotski y Luria 2007, 45). En esto, el psicólogo ruso y los estudios fenomenológicos, tal y como ahora veremos, coinciden plenamente. 


\section{LA PERCEPCIÓN EN LOS PLANTEAMIENTOS FENOMENOLÓGICOS}

Los estudios perceptivos fenomenológicos arrancan con el propio Husserl ${ }^{12}$ y se despliegan, a partir de sus trabajos, por los de sus innumerables prosélitos y seguidores. Con facilidad encontramos los trabajos del grupo de investigadores de Gotinga que, en línea husserliana, analizaron distintos aspectos de la percepción como el color, la profundidad, los elementos apriorísticos, el papel de las emociones. Hablamos de autores como Schapp, Jaensch, Katz, Hofmann, Pfänder o Scheler. ${ }^{13}$ Pero para nada se agotan en Gotinga los análisis fenomenológicos de la percepción. En posteriores generaciones hallamos nombres como los de Gurwitsch, Merleau-Ponty o Levinas y, aunque menos vinculado, pero gozando de mayor originalidad e interés para nuestro asunto, nuestro Ortega y Gasset, el primero en abordar los predicados culturales de la percepción dentro de esta corriente.

Pero más allá de enmarcar el conjunto de autores, nos interesa aquí remarcar las líneas generales del acercamiento de la fenomenología al estudio de la percepción para luego advertir las semejanzas con el trabajo vygotskiano, y este acercamiento se funda, precisamente, sobre el interés primigenio de la fenomenología por el estudio de las esencias. A la fenomenología le apremia el estudio de las esencias de toda cosa, pero comprendiendo a estas dentro de su existencia, a partir de su facticidad. ${ }^{14}$ Dado que es mediante la percepción -a través del cuerpo y los sentidos- que todo sujeto alcanza a tomar conciencia de ese mundo en el que se halla y desenvuelve, donde se despliega toda facticidad, la percepción resultará un aspecto ineludible para sus estudios. Es interesante que, como lo fuera para Vygotski, el entorno o mundo en el que el sujeto se desenvuelve y percibe cobra un peso substancial tanto para explicar el comportamiento humano, como para dar sentido al término mismo de lo que significa ser humano. No en vano, ese espacio donde las esencias se dan de manera fáctica fue llamado por Husserl "mundo de la vida" (Lebenswelt), el

\footnotetext{
12 Fundamentalmente: Husserl, Edmund. Investigaciones lógicas. Madrid: Alianza, 2014; y Husserl, Edmund. Ideas relativas a una fenomenología pura y una filosofía fenomenológica. México DF: FCE, UNAM, IIF, 2013.

${ }_{13}$ Sobre estos autores y su influencia en el campo de la fenomenología de la percepción ver: Montesó, Jorge. "Las fuentes orteguianas en su idea de 'atención'". Investigaciones fenomenológicas, núm. 12, 2015, pp. 137-156.

${ }^{14}$ Cfr. Merleau-Ponty, Maurice. Fenomenología de la percepción. Barcelona: Planeta, 1985, p. 7.
} 
mundo donde vivimos y que es vivido por nosotros, del que formamos parte y nos complementa, pues co-existimos en él y con él. Dado que ese mundo-dela-vida es, para el sujeto humano, un mundo en comunidad, forjado en el seno de una cultura, de tradiciones, el peso de la misma en nuestro desarrollo resultará decisivo. $Y$ no es menester enfatizar que dicha influencia se producirá, fundamentalmente, a través de la adquisición de conceptos, del lenguaje en tanto elemento nuclear de toda cultura. Así, es fácil advertir cuan cerca estamos de los postulados vygotskianos. Tal llega a ser el influjo del lenguaje para la fenomenología de la percepción que, para ellos, este alcanza a definir nuestra comprensión misma del mundo delimitando qué aspectos de la realidad alcanzaremos a atender (a percibir cognoscitivamente) y cuáles no. Como podría haber dicho Vygotski, aunque esta vez lo diremos en términos orteguianos, "si devolvemos a la palabra percepción su valor etimológico - donde se alude a coger, apresar- el concepto será el verdadero instrumento u órgano de la percepción y apresamiento de las cosas" (OC. I, 784). Es mediante conceptos que percibimos el mundo; y en semejante lógica: cada cultura será "un órgano de percepción distinto de todos los demás" (OC. II, 163), pues cada cultura, cada acervo conceptual, permitirá una visión del mundo, su Weltanschauung, el fruto de su propia perspectiva.

Como apuntábamos, hay tantos caminos como autores para abordar el estudio fenomenológico de la percepción, pero dado el objeto de nuestro artículo, interesados en rescatar el valor que la fenomenología da al lenguaje en el acto perceptivo, restringiremos el abanico a poco más de un par - siempre sin despistar la constante sombra husserliana que sobrevuela a cada uno de ellos-, para el caso hablamos de Schapp y de nuestro ya citado Ortega.

Sobre los trabajos de Schapp, aludiremos principalmente a su tesis Contribuciones a la fenomenología de la percepción. ${ }^{15}$ En ella, el germano aborda los elementos apriorísticos de la percepción, a saber, el papel que desempeñan las ideas o conceptos en tanto elementos directrices de nuestra atención para, con ellos, descubrir en el entorno los distintos objetos o elementos de la realidad. Schapp alude a la idea de claridad mental (Deutlichkeit) que se produce cuando hallamos, guiados por una idea preconcebida, ciertos elementos sensibles (co-

${ }^{15}$ Schapp, Wilhelm. Beiträge zur Phänomenologie der Wahrnehmung. Gotinga: M. Niemeyer, Halle/S, 1910. 
lor, luz, forma) en el entorno. Sin las ideas (o conceptos) que posibilitan la aprehensión de las cosas a través de su sentido, afirma Schapp que la percepción sería ciega, pues es guiados por ellas que otorgamos orden al caos de impresiones en que se nos presenta originalmente la realidad, y lo hacemos mediante la posibilidad que nos ofrecen para demarcar límites entre impresiones, para agruparlas en estructuras de significado descubriendo, así, los diferentes objetos de nuestro entorno o, como diría Vygotski, percibiendo objetos reales.

Schapp trata de explicar el funcionamiento de la percepción sensorial como un acto de interpretación, de atribución de sentido a las impresiones recibidas; impresiones que solo tras la aplicación del tamiz conceptual son comprehendidas, captadas. Las ideas, cual órganos de visión, posibilitan ese efecto de claridad mental que ofrece la captación de figuras, de objetos en el horizonte. De este modo, Schapp abre el camino a esas pre-visiones de las que nos hablará Ortega, necesarias para toda percepción cognoscitiva, en su caso motivadas por intereses y preferencias, en todo caso fundamentales para percibir en sentido pleno, pues de la realidad toda, solo alcanzaremos a percibir aquello de lo que ya tengamos cierta idea previa, cierta afinidad. Para ello, de un modo más o menos sentimental o cognoscitivo, deberán las cosas participar de nuestro proyecto de vida, de nuestra experiencia y, puesto que esta se despliega en el seno de una comunidad, lo harán a través de un lenguaje o patrimonio conceptual compartido.

En definitiva, lo que nos indica Schapp, y reafirmará Ortega, es que el lenguaje crea realidades y es generativo, y lo hace concediendo oportunidades al descubrimiento, mediante la percepción atenta, de fracciones de la realidad antes ignoradas, posibilitando nuevas perspectivas. Dijo Ortega en sus Meditaciones del Quijote: "cada concepto es literalmente un órgano con que captamos las cosas. Solo la visión mediante el concepto es una visión completa" (OC. I, 785). El acercamiento de Ortega a las ideas vygotskianas en este punto es indudable, como afirma de la Corte $(1997,100)$ : "Ortega esboza una idea de la adquisición del lenguaje muy cercana a la de Mead [...] y Vygotski [...]: la de una función comunicativa que acaba transformándose en una función puramente intelectual y de reestructuración del pensamiento", hasta el punto de determinar nuestra percepción del mundo y, con ella, nuestra comprensión plena del mismo. 
Quedándonos ya en Ortega, decía San Martín (cfr. 2012, 179) que la mayor aportación del madrileño a la fenomenología fue la de ofrecer una descripción sorprendentemente completa de las distintas capas o dimensiones que conforman la realidad en tanto vivida. Dicho a grandes trazos - pues adentrarnos en ello excedería el propósito del presente artículo ${ }^{16}$-, Ortega aprehende la realidad desde una doble dimensión: en tanto se nos da a los sentidos como impresión, desde lo sensible y superficial de las cosas, lo que designa como patente y podríamos expresar como "ver los árboles"; pero también desde el sentido, desde lo profundo o conceptual de la cosa percibida, que implica la red de conexiones y limites que conecta cada cosa con el resto, con el todo (cfr. OC. I, 749), es lo que Ilamaríamos lo latente, lo virtual o cultural: "el bosque". ${ }^{17}$ Lo que hacía de Ortega un pensador original era justamente que entendió que toda percepción del mundo, en esa síntesis o amalgama entre patente y latente, de lo impresionista y lo cultural, es "siempre percepción de algo enmarcado en un contexto vital desde el que lo percibido tiene su sentido" (San Martín 2012, 178), pues fuera de él nada sabemos. Dado que ese contexto vital en el que se desenvuelve el sujeto humano es siempre un contexto cultural, lo reseñable en la percepción humana será, precisamente, el conjunto de predicados que esta traiga consigo, en su caso, productos de la cultura en la que vive, predicados culturales. De aquí el acercamiento a Vygotski.

Cabe reseñar en este punto que, aunque salvando las distancias pues transitamos alegremente de una esfera perceptiva a otra lingüística, se halla cierto paralelismo entre la doble expresión de la realidad descrita por Ortega y la noción del habla vygotskiana. El ruso, en Pensamiento y lenguaje (cfr. 2007, 438), distingue entre dos planos del habla: el fonético y el semántico. No cuesta hallar importantes paralelismos entre el plano fonético del habla y el plano patente que describe Ortega; $y$, a su vez, otras tantas entre el plano semántico - vinculado al sentido, a lo profundo o relacional- con el latente. Del mismo modo, ambos coinciden en que dichas dimensiones, pese a la distinción, se dan

\footnotetext{
${ }^{16}$ La mejor descripción de esta doble estructura de la realidad la esboza Ortega en sus Meditaciones del Quijote. Para ampliar el tema, se puede consultar la obra citada de San Martín o también San Martín, Javier. Fenomenología y cultura en Ortega. Ensayos de Interpretación. Madrid: Tecnos, 1998.

${ }^{17}$ San Martín no duda en apostillar este gesto como la mayor aportación de Ortega a la fenomenología avanzando en años las críticas heideggerianas a Husserl sobre el "olvido" de los predicados culturales en su despliegue argumental sobre la percepción en Ideas. Cfr. San Martín, Javier. "La percepción como interpretación". Investigaciones fenomenológicas, núm. 6, 2008, pp. 13-32.
} 
íntimamente relacionadas entre sí, como si de un mismo proceso se tratase aunque a niveles o direcciones diferenciadas. No hablamos del mismo tipo de procesos, pero la similitud es, como poco, reseñable, ahora bien, tampoco casual. Ortega despliega esta distinción basándose en sus lecturas fenomenológicas y ya vimos, cuando repasamos el conocimiento que tenía Vygotski de los trabajos fenomenológicos, que la idea de que los dos planos del lenguaje forman una unidad proviene de las lecturas husserlianas, "una unidad que en términos de Husserl comprende los dos polos del acto psíquico total: el aspecto interno, significativo, semántico de la palabra; y el aspecto auditivo externo" (MacDonald 2000, 82).

Dicho lo cual, retomando la noción orteguiana de percepción, advertimos cómo su acento se volcó en la idea de "perspectiva". Para el madrileño, de la realidad toda, alcanzamos solo a conocer o percibir comprensiblemente una exigua fracción de la misma, la que nos permite nuestra perspectiva, tanto física como, en general, vital (de ahí que sea también cultural), pues esta perspectiva, posibilitada por nuestra atención, vendrá especialmente influenciada por el contexto cultural en el que el sujeto viva. ${ }^{18}$ Veámoslo con detenimiento.

La realidad en la que vivimos, decía Ortega, es una y la misma para todos, pero sucede que, de ella, cada cual acaba obteniendo su propia fracción, su propio paisaje. Esto no ocurre porque la realidad sea diferente para cada sujeto, ni porque este ejerza algún tipo de elaboración constructiva sobre la realidad percibida. Nuestro conocimiento, indica Ortega, no es una cuestión ni de la realidad - como afirmaba el realismo- ni del sujeto - que defendía el idealismo-, sino del modo en que ambos correlacionan, esto es, de perspectiva, de selección. Nuestro conocimiento de la realidad, afirma, vendrá marcado por un proceso de selección, un proceso de filtrado atencional mediante el que desplegar nuestra perspectiva, donde la cultura, a través del lenguaje, ejercerá un peso fundamental. Recordemos que el mismo Vygotski estudió la percepción humana a través de los procesos de selección.

El proceso de selección, para Ortega, es un proceso de doble disposición. ${ }^{19}$ En él se produce un primer filtrado que bien podríamos calificar de fisiológico,

\footnotetext{
${ }^{18}$ Sobre cómo los cambios culturales en un mismo pueblo modifican la perspectiva del sujeto en tanto espectador: Las Atlántidas (OC. III, 743) y "Sobre el punto de vista en las artes" (OC. V, 160).

19 Cfr. "Biología y pedagogía" (OC. II, 399) o "Dios a la vista" (OC. II, 605).
} 
compartido por toda la especie y que vendrá determinado por la complexión de nuestros sentidos y cerebro ( $v$. g. los humanos no alcanzamos a percibir radiaciones ultravioletas o infrarrojas). Este proceso bien armonizaría con la idea de función elemental vygotskiana. Pues bien, por él decía Ortega que: "cada especie, merced de su sensibilidad, selecciona del mundo infinito un repertorio de objetos, únicos que para el animal existirán, y articulados en admirable arquitectura, formarán su contorno. Hay un mundo para el hombre y otro para el águila, y otro para la araña" (OC. II, 422-423).

Sobre este filtrado elemental se produce otro más sofisticado, "más complejo". Este será un filtrado ya no orgánico, sino proveniente de nuestra intimidad, de nuestra biografía o experiencia, motivado por preferencias e intereses, resultado de una elección que, en consecuencia, implicará en su manifestación sentimientos, creencias, valores, tradiciones. En definitiva, hablamos de un filtrado cultural en la base del cual se hallará, como fundamento de todo predicado, el uso de signos, los conceptos que el sujeto, en tanto miembro de una comunidad, emplee.

La cercanía con los postulados vygotskianos es palmaria, tanto que también Ortega llegará a afirmar, jugando entre el ver y el mirar, que, como humanos "no podemos ver sin mirar" (OC. II, 605-606). Cierto que Ortega va más allá en sus determinaciones de lo aquí visto en Vygotski, ${ }^{20}$ pues el ruso se centra más en el papel del lenguaje, en cambio Ortega apela a una percepción culturalmente mediada entendiendo "cultura" en sentido más amplio, implicando en su seno las disposiciones del espíritu, las emociones, lo biográfico en general, pues lo que en el fondo se implica es la vida en su conjunto. Evidentemente, el fundamento de esa determinación gravita sobre el uso de signos, de ahí que Ortega hable siempre de una percepción cultural, porque en el ejercicio perceptivo, cada cultura, cada pueblo, mediante sus conceptos, cederá a sus miem-

\footnotetext{
20 iAunque quizá no tanto! Llega a decir Vygotski que "para comprender el habla ajena, nunca es suficiente comprender solo algunas palabras: debemos comprender el pensamiento del interlocutor. Pero incluso la comprensión del pensamiento del interlocutor, si no se comprende su motivo, aquello por lo cual expresa el pensamiento, resulta una comprensión incompleta" $(2007,510)$, necesitamos de los motivos, de los intereses. Vygotski estudiará el papel de las emociones a partir de los años 30. En 1934, en la conferencia El problema del entorno - cabe decir que su transcripción es muy cuestionada- hace referencia a la "experiencia emocional" mediante el término ruso perezhivanie. Van der Veer y Valsiner (1994, 354) defienden que perezhivanie "sirve para expresar la idea de que una y la misma situación objetiva puede ser interpretada, percibida, experimentada o vivida por diferentes niños de modo diferente. $\mathrm{Ni}$ la 'experiencia emocional' [...], ni 'interpretación' [...] son traducciones totalmente adecuadas del sustantivo. Su significado está íntimamente ligado al verbo alemán 'erleben' (cf. 'Erlebnis', 'Erlebte Wirklichkeit')". Las semejanzas con la fenomenología son más que notables.
} 
bros un modo particular de dar sentido a las cosas, pues, en términos vygotskianos, la palabra "es la expresión directa de la naturaleza histórica de la conciencia humana" (Vygotski 2007, 515).

Como vemos, las semejanzas entre Vygotski y Ortega son perspicuas. Pero no concluyen aquí. También Ortega se vistió de filogenetista, como lo hiciese Vygotski, y lo hizo para hablarnos de evolución humana, esta vez, en términos perceptivos. Así, Ortega analizó cómo el sujeto pasa, en su evolución, por tres etapas que, según el grado de control atencional del que se disponga, dividen su curso entre un ser que inicialmente vive "alterado" hasta, tras obtener la capacidad de "ensimismarse", convertirse en un ser que retorna al mundo conocedor de su intimidad y capaz de trazar una serie de planes de acción con los que dominar su circunstancia, ${ }^{21}$ a saber, "con un sí mismo que antes no tenía" (OC. X, 145). Establecía Ortega, usando como parámetro la dirección que la atención toma en cada momento (centrífuga o centrípeta), un horizonte desde el que contemplar todo el continuum de complejidad progresiva desde una percepción "alterada" o gestionada por el entorno, hasta alcanzar un respetable grado de complejidad cuando la atención se orienta voluntariamente a la tarea, pretendiendo su óptima ejecución, esto es, hasta que provoca que todo nuestro ser se disponga frente a lo otro desde una actitud cuidadosa e intencionada.

Este itinerario progresivo se alcanza tras un laborioso proceso de adiestramiento de la atención para conseguir, en un determinado momento de madurez cognitiva, la capacidad atender a nuestra intimidad - de re-flexionar-, de poder ensimismarse y generar un instante de abstracción en el que planificar, mediante el pensamiento, toda acción futura - "el pensamiento no es un don del hombre, sino adquisición laboriosa" (OC. X, 150)-. Se repite, de algún modo, el esquema vygotskiano que ve en el animal a un ser que vincula percepción a acción de un modo franco para situar, en sus antípodas, al hombre contemporáneo, adulto, en quien el esquema percepción-lenguaje-acción se despliega con plenitud. No en vano definía Ortega la acción como un "actuar sobre el contorno de las cosas materiales o de los otros hombres conforme a un plan preconcebido en una previa contemplación o pensamiento" (OC. X, 151). Como

\footnotetext{
${ }^{21}$ Sobre ello: Ensimismamiento y alteración. Meditación de la técnica (OC. V, 529); "El mito del hombre allende la técnica" (OC. VI, 811); Ideas y Creencias (OC. V, 672); Lecciones VII y VIII de ¿Qué es filosofía? (OC. VIII, 235 y sig.); o Sobre ensimismarse y alterarse (OC. V, 251).
} 
bien compartiría Vygotski, y con ello finalizamos nuestra alígera comparativa, ofrecía Ortega un horizonte filogenético donde el hombre se daba como una laboriosa excepción dentro del reino animal, aceptando como momento crítico esa posibilidad de torcer la atención y reflexionar, un gesto posibilitado, entre otros factores de maduración, por la capacidad de usar signos. Esta idea, armonizada con la comprensión ontológica ya revisada, demuestran hasta qué punto las ideas de Ortega, de los estudios perceptivos de la fenomenología en general, pueden llegar a entroncar con los postulados vygotskianos aquí desplegados.

\section{BIBLIOGRAFÍA}

DE LA CORTE, Luís. "Acción y convivencia: algunas implicaciones del pensamiento de Ortega y Gasset para la psicología social y las ciencias humanas". Revista de historia de la psicología, núm. 18, vol. 1-2, 1997, pp. 97-102.

García, Luciano. N. "La 'canonización' de Vigotski en España y Argentina (19781991)". Revista de historia de la psicología, núm. 36, vol. 2, 2015, pp. 7-30.

Holenstein, Elmar. "Jakobson and Husserl". Human Context, núm. 7, 1975, pp. 6183.

HUSSERL, Edmund. La filosofía como ciencia estricta. Buenos Aires: Terramar, 2007.

- Ideas relativas a una fenomenología pura y una filosofía fenomenológica. México DF: FCE, 2013.

- Investigaciones lógicas. Madrid: Alianza, 2014.

KÖHLER, Wolfgang. "Zur Psychologie des Schimpansen". Aus der Anthropoidenstation auf Teneriffa, 5, Sitzungsberichte der Preußischen Akademie der Wissenschaften, 1921.

KLYUKANOV, Igor E. "La fenomenología en el espejo de la psicolingüística: una perspectiva vygotskiana". Contrastes. Revista interdisciplinar de filosofía, núm. 1, 1996, pp. 319-327.

MACDONALD, Paul. "Phenomenological factors in Vygotsky's mature psychology". History of the Human Sciences, núm. 13, vol. 3, 2000, pp. 69-93.

MERLEAU-PONTY, Maurice. Fenomenología de la percepción. Barcelona: Planeta, 1985. MONTESÓ, Jorge. "Las fuentes orteguianas en su idea de 'atención'". Investigaciones fenomenológicas, núm. 12, 2015, pp. 137-156. 
PERINAT, Adolfo. "La teoría histórico-cultural de Vygotsky: algunas acotaciones a su origen y su alcance". Revista de Historia de la Psicología, núm. 28, vol. 2/3, 2007, pp. 19-25.

- "Mis desencuentros y mi reencuentro con Vygotsky". Quaderns de Psicologia, núm. 11, vol. 1/2, 2009, pp. 51-60.

ORTEGA y GASSET. José. Obras Completas, Madrid: Taurus, 2004-2010.

SAN MARTÍN, Javier. Fenomenología y cultura en Ortega. Ensayos de Interpretación. Madrid: Tecnos, 1998.

- "La percepción como interpretación". Investigaciones fenomenológicas, núm. 6, 2008, pp. 13-32.

- La fenomenología de Ortega y Gasset. Madrid: Biblioteca Nueva, 2012.

SchAPP, Wilhelm. Beiträge zur Phänomenologie der Wahrnehmung. Gotinga: M. Niemeyer, Halle/S., 1910.

SiguÁn, Miguel. "Relectura de Luria". Revista de historia de la psicología, núm. 33, vol. 1, 2010, pp. 51-64.

VAN der Veer, René. Lev Vygotsky. Londres: Continuum Intr. Publishing G., 2007.

- Vygotsky in context: 1900-1935. En DANIELS, H., COLE, M. y WERTCH, J. V. (Eds.). The Cambridge Companion to Vygotsky. Nueva York: Cambridge University Press, 2007, pp. 21-49.

- y VALSINER, Jaan. (Eds.). The Vygotsky reader. Oxford: Blackwell, 1994.

- y YASNITSKY, Anton. "Vygotsky in English: What still needs to be done". Integrative Psychological and Behavioral Science, núm. 45, vol. 4, 2011, pp. 475- 493.

- "Vygotsky the published. Who wrote Vygotsky and what Vygotsky actually wrote". En YASNITSKY, A. y VAN der VeER, R. (Eds.) Revisionist Revolution in Vygotsky Studies. Londres: Routledge, 2016, pp. 73-93.

- VV.AA. Actualidad de Lev. S. Vygotski. Siguán, M. (coord.). Barcelona: Anthropos, 1987.

- VYGotski, Lev S. "El método de investigación reflexológica y psicológica". Problemas de la psicología contemporánea, núm. 2, 1930, pp. 26-46.

- Collected Works. vol. 3, Problems of the theory and history of psychology. Including the chapter on the crisis in psychology. En RIEBER, T. W. y WOLLOCH, J. (Eds.). Nueva York y Londres: Plenum Press, 1987.

- Pensamiento y habla. Buenos Aires: Colihue, 2007. 
- VygotSki, Lev S. y LURIA, Alexander R. El instrumento y el signo en el desarrollo del niño. Madrid: Fundación Infancia y aprendizaje, 2007.

YASNITSKY, Anton. "A transnational history of "the beginning of a beautiful friendship"the birth of the cultural-historical Gestalt psychology of Alexander Luria, Kurt Lewin, Lev Vygotsky, and others". En YASNITSKY, A. y VAN der VeER, R. (Eds.) Revisionist Revolution in Vygotsky Studies. Londres: Routledge, 2016, pp. 201-226.

YASNITSKY, Anton y VAN der VEER, René. Revisionist Revolution in Vygotsky Studies. Londres: Routledge, 2016.

ZaVershneva, Ekaterina. "The Vygotsky Family Archive: New Findings. Notebooks, Notes, and Scientific Journals of L.S. Vygotsky (1912-1934)". Journal of the Russian and East European Psychology, núm. 48, vol. 1, 2010. 